\title{
Divergence of perturbation theory: Steps towards a convergent series
}

\author{
Sergio A. Pernice \\ Department of Physics and Astronomy, University of Rochester, Rochester, New York 14627 \\ Gerardo Oleaga \\ Departamento de Matematica Aplicada, Facultad de Matematicas, Universidad Complutense, 28040 Madrid, Spain
}

(Received 14 August 1996; published 24 December 1997)

\begin{abstract}
The mechanism underlying the divergence of perturbation theory is exposed. This is done through a detailed study of the violation of the hypothesis of Lebesgue's dominated convergence theorem using familiar techniques of quantum field theory. That theorem governs the validity (or lack of it) of the formal manipulations done to generate the perturbative series in the functional integral formalism. The aspects of the perturbative series that need to be modified to obtain a convergent series are presented. Useful tools for a practical implementation of these modifications are developed. Some resummation methods are analyzed in the light of the above mentioned mechanism. [S0556-2821(97)04424-X]
\end{abstract}

PACS number(s): 11.10.Jj

\section{INTRODUCTION}

A typical quantity used to analyze the nature of the perturbative expansion in quantum field theory is the partition function

$$
Z(\lambda)=\frac{1}{Z_{0}} \int[d \phi] e^{-S[\phi]},
$$

with

$$
S[\phi]=\int d^{d} x\left[\frac{1}{2}\left(\partial_{\mu} \phi\right)^{2}+\frac{1}{2} m^{2} \phi^{2}+\frac{\lambda}{4} \phi^{4}\right] .
$$

The normalization factor $1 / Z_{0}$ is the partition function of the free field $(Z \rightarrow 1$ when $\lambda \rightarrow 0)$. The analysis of the perturbative expansion of any Green's function goes along similar lines to that for $Z$. In the example above we consider a scalar field theory for simplicity.

The traditional argument for understanding the divergent nature of the perturbative expansion can be traced back to Dyson [1]. Although the form was different, the content of his argument is captured by the following statement: "If the perturbative series were to converge to the exact result, the function being expanded would be analytic in $\lambda$ at $\lambda=0$. But the function ( $Z$ for example) is not analytic in $\lambda$ at that value. Therefore, as a function of $\lambda$, the perturbative series is either divergent or converges to the wrong answer."

Estimates of the large order behavior of the coefficients of the perturbative series showed that the first possibility is the one actually realized $[2,3]$. That $Z$, as a function of $\lambda$, is not analytic at $\lambda=0$ can be guessed by simply noting that if in its functional integral representation [Eq. (1)] we make the real part of $\lambda$ negative (though arbitrarily small), the integral diverges. In fact, there is a branch cut in the first Riemann sheet that can be chosen to lie along the negative real axis, extending from $\lambda=-\infty$ to $\lambda=0[4,5]$.

The above argument is very powerful and extends to the perturbative series of almost all other nontrivial field theories. It has also motivated a series of very important calcu- lations of the large order behavior of the perturbative coefficients [2] and general analysis of the structure of field theories [6], as well as improvements over perturbative computations of different physical quantities [2].

For all its power, it is fair to say that this argument, as is typically the case with a reductio ad absurdum type of argument, fails to point towards a solution of the problem of divergence. It is only through the indirect formalism of Borel transforms that questions of the recovery of the full theory from its perturbative series can be discussed $[5,7,8]$.

In this paper an alternative way of understanding the divergent nature of the perturbative series is presented. This way of understanding the problem complements the traditional argument briefly described above, hopefully illuminating aspects that the traditional approach leaves obscure. In particular, as we will see, the arguments in this paper point directly towards the aspects of the perturbative series that need to be modified to achieve a convergent series. It is hoped that the way of understanding the problem presented here will help to provide new insights into the urgent problem of extracting nonperturbative information out of quantum field theories.

In Sec. II we develop our analysis of the divergence of perturbation theory. In Sec. III we point out the ingredients that, according to the analysis of Sec. II, a modification of perturbation theory would need to achieve convergence. We also present a remarkable formula (64) that allows us to implement such modifications in terms of Gaussian integrals, paving the way for the application of this convergent modified perturbative series to quantum field theories. The proof of the properties of the function (64) is given in Appendix A. In Sec. IV we analyze recent work on the convergence of various optimized expansions [9-16] in terms of the ideas presented here. In Sec. V we summarize our results and mention directions of the work currently in preparation. Finally, in Appendix B, we apply the ideas of this paper in a simple but illuminating example for which we actually develop a convergent series by modifying the aspects of the perturbative series pointed out by our analysis as the source of divergence. 


\section{LEBESGUE'S DOMINATED CONVERGENCE THEOREM AND PERTURBATION THEORY}

\section{A. Wrong step in perturbation theory}

Although the notation will not always be explicit, we will work in an Euclidean space of dimension smaller than 4 and in a finite volume.

Let us remember how the perturbative series is generated in the functional integral formalism for a quantity like $Z$ :

$$
\begin{aligned}
Z(\lambda) & =\int[d \phi] \exp \left(-\int d^{d} x\left[\frac{1}{2}\left(\partial_{\mu} \phi\right)^{2}+\frac{1}{2} m^{2} \phi^{2}\right]-\frac{\lambda}{4} \int d^{d} x \phi^{4}\right) \\
& =\int[d \phi] \sum_{n=0}^{\infty} \frac{(-1)^{n}}{n !}\left(\frac{\lambda}{4} \int d^{d} x \phi^{4}\right)^{n} \exp \left(-\int d^{d} x\left[\frac{1}{2}\left(\partial_{\mu} \phi\right)^{2}+\frac{1}{2} m^{2} \phi^{2}\right]\right) \\
& =\sum_{n=0}^{\infty} \int[d \phi] \frac{(-1)^{n}}{n !}\left(\frac{\lambda}{4} \int d^{d} x \phi^{4}\right)^{n} \exp \left(-\int d^{d} x\left[\frac{1}{2}\left(\partial_{\mu} \phi\right)^{2}+\frac{1}{2} m^{2} \phi^{2}\right]\right) .
\end{aligned}
$$

The final sum is in practice truncated at some finite order $N$. The functional integrals that give the contribution of every order $n$ are calculated using Wick's theorem and Feynman's diagram techniques with the corresponding renormalization.

We see then that the generation of the perturbative series in the functional integral formalism is a two-step process. First Eq. (4), the integrand, is expanded in powers of the coupling constant, and then Eq. (5), the sum, is interchanged with the integral. ${ }^{1}$

It will be convenient to have a simpler example in which the arguments of this paper become very transparent. Consider the simple integral

$$
z(\lambda)=\frac{1}{\sqrt{\pi}} \int_{-\infty}^{\infty} d x e^{-\left[x^{2}+(\lambda / 4) x^{4}\right]}
$$

and its corresponding perturbative expansion

$$
\begin{aligned}
z(\lambda) & =\frac{1}{\sqrt{\pi}} \int_{-\infty}^{\infty} d x \sum_{n=0}^{\infty} \frac{(-1)^{n}}{n !}\left(\frac{\lambda}{4} x^{4}\right)^{n} e^{-x^{2}} \\
& =\frac{1}{\sqrt{\pi}} \sum_{n=0}^{\infty} \int_{-\infty}^{\infty} d x \frac{(-1)^{n}}{n !}\left(\frac{\lambda}{4} x^{4}\right)^{n} e^{-x^{2}} \\
& \equiv \sum_{n=0}^{\infty}(-1)^{n} c_{n} \lambda^{n} .
\end{aligned}
$$

This simple integral has been used many times in the past as a paradigmatic example of the divergence of perturbation

\footnotetext{
${ }^{1}$ In this paper we will often use the familiar word "integrand" to refer to $e^{-S}$ or any functional inside the functional integration symbol. It would be more precise to preserve this word for $e^{-S_{\text {int }}}$ in the measure defined by the free field. The terminology used here is, however, common practice in the quantum field theory literature and also helps to emphasize the similarities with the intuitive finite dimensional case presented below.
}

theory [8]. It is therefore especially well suited for a comparison between the traditional arguments and the ones presented in this paper.

Again we use the two-step process to generate the perturbative series. First the integrand is expanded in powers of $\lambda$, Eq. (7), and then the sum is interchanged with the integral (8). In this simple example the perturbative coefficients can be calculated exactly for arbitrary $n$. In the large $n$ limit they become

$$
c_{n} \sim \sqrt{\frac{2}{2 \pi}}(n-1) ! \quad \text { when } n \rightarrow \infty
$$

With such factorial behavior, the series diverges for all $\lambda$ different from zero as is well known. On the other hand, the function $z(\lambda)$, as defined in Eq. (6), gives a well-defined positive real number for every positive real $\lambda$. Therefore one or both of the two steps done to generate the perturbative series must be wrong.

Similarly, in the functional integral case normalized with respect to the free field (1), $Z$ is a well-defined number while its perturbative series diverges. Again, one or both of the two steps must be wrong.

The first step, the expansion of the integrand in powers of $\lambda$, is clearly correct. As the integrand (not the integral) is analytic in $\lambda$ for every finite $\lambda$, the expansion merely corresponds to a Taylor series. The second step, the interchange of sum and integral, must therefore be the wrong one.

The next obvious step is then to recall the theorems that govern the interchange between sums and integrals in order to understand in detail why this step is wrong in our case. The most powerful theorem for this purpose is Lebesgue's well-known theorem of dominated convergence. In a simplified version, sufficient for our purposes, it says the following.

Let $f_{N}$ be a sequence of integrable functions that converge pointwisely to a function $f$,

$$
f_{N} \rightarrow f \quad \text { as } N \rightarrow \infty
$$

and bounded in absolute value by a positive integrable function $h$ (dominated): 


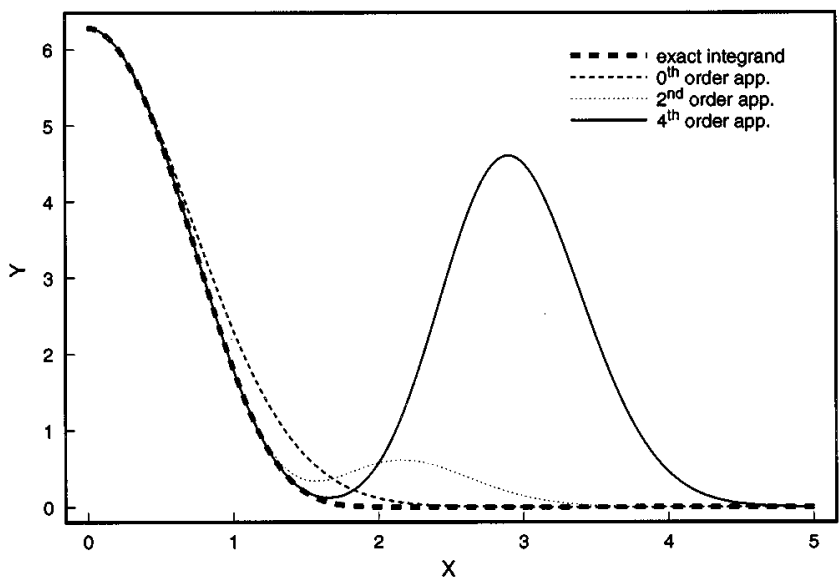

FIG. 1. Exact integrand, zeroth, second, and fourth perturbative approximations. $\lambda=1$.

$$
\left|f_{N}\right| \leqslant h \quad, \quad \forall N
$$

Then, it is true that

$$
\lim _{N \rightarrow \infty} \int f_{N}=\int \lim _{N \rightarrow \infty} f_{N}=\int f .
$$

As a special case, if the convergence (11) is uniform and the measure of integration is finite, then the interchange is also valid. It should be emphasized that Lebesgue's theorem follows from the axioms of abstract measure theory. Therefore if the problem under consideration involves a well-defined measure, as is the case for the quantum field theories considered here [16], the theorem holds.

In our case we can write, formally, ${ }^{2}$

$$
\begin{aligned}
f_{N}[\phi(x)]= & \frac{1}{Z_{0}} \sum_{n=0}^{N} \frac{(-1)^{n}}{n !}\left(\frac{\lambda}{4} \int d^{d} x \phi^{4}\right)^{n} \\
& \times \exp \left(-\int d^{d} x\left[\frac{1}{2}\left(\partial_{\mu} \phi\right)^{2}+\frac{1}{2} m^{2} \phi^{2}\right]\right)
\end{aligned}
$$

for the functional integral case and

$$
f_{N}(x)=\frac{1}{\sqrt{\pi}} \sum_{n=0}^{N} \frac{(-1)^{n}}{n !}\left(\frac{\lambda}{4} x^{4}\right)^{n} e^{-x^{2}}
$$

for the simple integral example.

One important aspect of the dominated convergence theorem approach to analyze the divergence of perturbation theory is that it focuses on the integrands, objects that are relatively simple to analyze. On the contrary, the analyticity approach briefly described in the Introduction focuses on the integrals which are much more difficult to analyze. So before we try to understand the aspects of the dominated convergence theorem that fail in our case, it will be useful to study some properties of the integrand for the intuitive simple example. In Fig. 1, the exact integrand, together with some perturbative approximations, are displayed. We can appreci-

\footnotetext{
${ }^{2}$ See previous footnote.
}

ate the way in which the successive approximations behave. For small $x$, and up to some critical value that we call $x_{c, N}$ (where the subindex $c$ stands for critical while the subindex $N$ indicates that this value changes with the order) the perturbative integrands approximate the exact integrand very well. Even more, $x_{c, N}$ grows with $N$. But for $x$ bigger than $x_{c, N}$ a "bump" begins to emerge. The height of these bumps, as we will see in detail shortly, grows factorially with the order, while the width remains approximately constant. So the larger the order in perturbation theory, the larger the region in which the perturbative integrands approximate the exact integrand very well, but the stronger the upcoming deviation. As we will see shortly, it is precisely this deviation that is responsible for the divergence of the perturbative series and the famous factorial growth. We will also see that an exactly analogous phenomenon happens in the functional integral case and is again responsible for the divergence of the perturbative series.

Returning to the problem of understanding the aspects of the dominated convergence theorem that fail in the perturbative series, we will now show that the sequence of integrands of Eq. (14) and Eq. (15) converges, respectively, to the exact integrands

$$
F=\frac{1}{Z_{0}} \exp \left(-\int d^{d} x\left[\frac{1}{2}\left(\partial_{\mu} \phi\right)^{2}+\frac{1}{2} m^{2} \phi^{2}\right]-\frac{\lambda}{4} \int d^{d} x \phi^{4}\right)
$$

and

$$
f=\frac{1}{\sqrt{\pi}} e^{-\left[x^{2}+(\lambda / 4) x^{4}\right]},
$$

but not in a dominated way. That is, there is no positive integrable function $h$ that satisfies Eq. (12).

\section{B. Failure of domination in the simple example}

That the sequence of integrands of Eq. (14) and Eq. (15) converges, respectively, to the exact integrands (16) and (17) is obvious, since, as mentioned before, for finite $\lambda$ they are analytic functions of $\lambda$ and so their Taylor expansions converge (at least for finite field strength). To see the failure of the domination hypothesis it is convenient to analyze the "shape" of every term of $f_{N}$. Namely, for the field theory case,

$$
\begin{aligned}
c_{n}[\phi(x)] \equiv & \frac{1}{Z_{0}} \frac{(-1)^{n}}{n !} \frac{1}{4^{n}}\left(\lambda \int d^{d} x \phi^{4}\right)^{n} \\
& \times \exp \left(-\int d^{d} x\left[\frac{1}{2}\left(\partial_{\mu} \phi\right)^{2}+\frac{1}{2} m^{2} \phi^{2}\right]\right),
\end{aligned}
$$

while for the simple integrand

$$
c_{n}(x)=\frac{1}{\sqrt{\pi}} \frac{(-1)^{n}}{n !}\left(\frac{\lambda}{4}\right)^{n} x^{4 n} e^{-x^{2}} .
$$

In this section we analyze the failure of the domination hypothesis for the simple example (6) because it turns out to be 


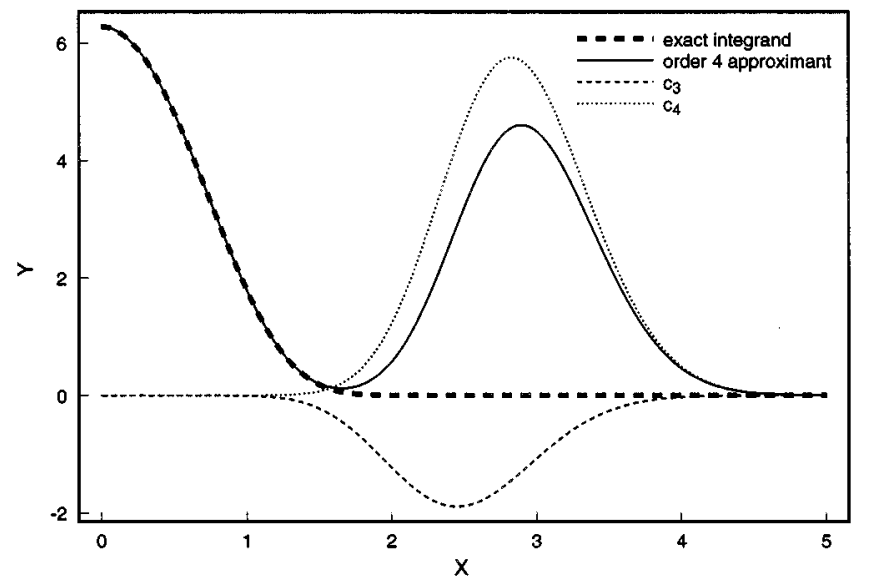

FIG. 2. Exact integrand and fourth perturbative approximation together with the third and fourth terms. $\lambda=1$.

remarkably similar to the field theory example analyzed in the next section. In Fig. 2 we can examine the functions $c_{3}(x)$ and $c_{4}(x)$ for $\lambda=1$ corresponding to the simple integrand case that we analyze first. The maximum of $c_{n}(x)$ is reached at

$$
x_{\max }= \pm(2 n)^{1 / 2} \text {. }
$$

There, for large $n$, the function takes the value

$$
c_{n}\left(x_{\max }\right)=\frac{1}{2 \pi^{3 / 2}}(-1)^{n}(n-1) ! \lambda^{n} .
$$

On the other hand, the width remains constant as $n$ increases as can be seen by a Gaussian approximation around the maximum $x_{\max }=(2 n)^{1 / 2}$ :

$$
c_{n}(x) \approx \frac{1}{2 \pi^{3 / 2}}(-1)^{n}(n-1) ! \exp \left\{-2\left[x-(2 n)^{1 / 2}\right]^{2}\right\} \lambda^{n} \text {. }
$$

The integration of this Gaussian approximation gives, for large $n$,

$$
\int d x c_{n}(x) \approx \frac{1}{2} \frac{\sqrt{2}}{2 \pi}(-1)^{n}(n-1) ! \lambda^{n}
$$

in accordance with Eq. (10) if we take into account the factor of 2 coming from the two maxima $\pm(2 n)^{1 / 2}$.

The mechanism of convergence of the $f_{N}$ 's to $f$ now becomes clear. The $f_{N}$ 's are made out of a pure Gaussian (the "free" term) plus "bumps" (the perturbative corrections) that alternate in sign (see Fig. 2). The height of these bumps grows factorially with the order, while their width remains approximately constant. More specifically, the Gaussian approximation around the maxima [Eq. (22)], which becomes exact when the order goes to infinity, has a variance independent of the order. For fixed $N$ and for $x$ smaller than a certain value, the bumps exhibit a delicate near-cancellation, leaving only a small remnant that modifies the free integrand into the interacting one. However, for $x$ larger than that value, the last bump begins to emerge and, being the last, does not have a successor to cancel it (in the $N \rightarrow \infty$ limit, there is no last bump and the convergence is achieved for every $x$ ). Consequently, beyond a certain value $x_{c, N}$, the function $f_{N}$ deviates strongly from $f$ and is governed by the uncanceled $N$ th bump, with height proportional to $(N-1)$ ! and finite variance. This is so because, since the height of the bump grows factorially with the order, for $N$ large enough the last bump is far greater than all the previous ones and remains almost completely uncanceled. Furthermore, since the variance of the bumps is independent of the order, this means that for every finite order, there is a region of finite measure in which the perturbative integrand is of the order of the height of the last bump. In Fig. 2 we can see how the function $c_{4}(x)$ is left almost completely uncanceled by $c_{3}(x)$ and dominates the deviation of $f_{4}$ from $f$.

That $x_{c, N}$ (the value of $|x|$ up to which the perturbative integrand very accurately approximates the exact one) grows with $N$, going to infinity when $N \rightarrow \infty$, is a simple consequence of Taylor's theorem applied to the analytic function $e^{-\lambda x^{4} / 4}$

The above analysis makes clear the failure of the domination of the sequence of Eq. (15) towards $f$ [Eq. (17)]. Indeed, any positive function $h(x)$ with the property

$$
\left|f_{N}(x)\right| \leqslant h(x), \quad \forall N
$$

fails to be integrable, since it has to "cover" the bump, whose area grows factorially with $N$. Therefore, although the sequence of $f_{N}(x)$ 's converges to $f(x)$, the convergence is not dominated, as we wanted to show.

Equation (23), together with the above comments, indicates that the same reason for which the sequence of integrands (19) fails to be dominated is the one that produces the factorial growth in the perturbative series.

In the field theory case, although we cannot rely on figures such as Eqs. (1) and (2) to guide our intuition, we will see that the analogy with the simple integral example is so close that the interpretation is equally transparent.

\section{Failure of domination in quantum field theory}

For quantum field theory, as for the simple example analyzed above, it is convenient to consider every term $c_{n}[\phi(x)]\left[\right.$ Eq. (18)] of the perturbative approximation $f_{N}$ [Eq. (14)] to the exact integrand [Eq. (16)],

$$
\begin{aligned}
c_{n}[\phi(x)]= & \frac{1}{Z_{0}} \frac{(-1)^{n}}{n !} \exp \left(-\int d^{d} x\left[\frac{1}{2}\left(\partial_{\mu} \phi\right)^{2}+\frac{1}{2} m^{2} \phi^{2}\right]\right. \\
& \left.+n \ln \left[(\lambda / 4) \int d^{d} x \phi^{4}\right]\right)
\end{aligned}
$$

where we have written the $n$th power of the interaction in exponential form. The mathematical analysis below follows closely the discussions in Chap. 38 of Ref. [8]. Although the problem treated there is different from the one treated here, many techniques used in [8] can be directly applied here.

For $n$ large enough, the analysis of its "shape" reduces to the familiar procedure of finding its maxima, as in the case of the simple integrand. The equation determining the 
maxima of $c_{n}[\phi(x)]$ is the equation that minimizes the exponent, and can be thought of as the equation of motion of the effective action

$$
S[\phi]=\int d^{d} x\left[\frac{1}{2}\left(\partial_{\mu} \phi\right)^{2}+\frac{1}{2} m^{2} \phi^{2}\right]-n \ln \left[\frac{\lambda}{4} \int d^{d} x \phi^{4}\right],
$$

which is

$$
-\nabla^{2} \phi+m^{2} \phi-\frac{4 n}{\int d^{d} x \phi^{4}} \phi^{3}=0 .
$$

Making the change of variables

$$
\begin{aligned}
\phi(x) & =m\left(\frac{\int d^{d} x \phi^{4}}{4 n}\right)^{1 / 2} \varphi(m x) \\
& =m^{d / 2-1}\left(\frac{4 n}{\int d^{d} u \varphi^{4}(u)}\right)^{1 / 2} \varphi(m x),
\end{aligned}
$$

we find that $\varphi$ satisfies the equation

$$
-\nabla^{2} \varphi(u)+\varphi(u)-\varphi^{3}(u)=0, \quad u \equiv m x .
$$

This equation corresponds to the instanton equation of the negative mass $\lambda \phi^{4}$ theory. The analysis of its solutions can be found in many places. We are interested in solutions with minimal, finite action. For these solutions, in the infinite volume limit, scaling arguments provide very interesting information. We mentioned at the beginning of Sec. II A that we work in a finite volume. However, if the volume is large enough, the infinite volume arguments used below remain valid up to errors that go to zero exponentially fast when the volume goes to infinity.

Since the solution $\phi_{\max }(x)$ (the subindex "max" indicates that, in functional space, $c_{n}[\phi(x)]$ reaches its maximum at $\phi_{\max }(x)$; this should not be confused with the fact that the the action (26) reaches its minimum there) is a minimum of the action (26), then given an arbitrary constant $\alpha$, $S\left[\alpha \phi_{\max }(x)\right]$ should have a minimum at $\alpha=1[8,17]$. This implies the equation

$$
\int d^{d} x\left(\partial_{\mu} \phi_{\max }\right)^{2}+m^{2} \int d^{d} x \phi_{\max }^{2}-4 n=0 .
$$

Similarly, $S\left[\phi_{\max }(\alpha x)\right]$ should also have a minimum at $\alpha=1$, implying

$$
\frac{(2-d)}{d} \int d^{d} x\left(\partial_{\mu} \phi_{\max }\right)^{2}-m^{2} \int d^{d} x \phi_{\max }^{2}+2 n=0 .
$$

Solving the system of equations (30) and (31) we obtain

$$
\begin{gathered}
\int d^{d} x\left(\partial_{\mu} \phi_{\max }\right)^{2}=n d, \\
m^{2} \int d^{d} x \phi_{\max }^{2}=n(4-d),
\end{gathered}
$$

from which we conclude in particular that the integral

$$
\int d^{d} x\left[\frac{1}{2}\left(\partial_{\mu} \phi_{\max }\right)^{2}+\frac{1}{2} m^{2} \phi_{\max }^{2}\right]=2 n
$$

is independent of the dimension. The relations (32) and (33) can be explicitly checked in the case $d=1$ (quantum mechanics), in which the solutions to Eq. (27) are known analytically. They are

$$
\phi_{\max }^{d=1}(t)=\left(\frac{3 n}{2 m}\right)^{1 / 2} \frac{1}{\cosh \left[m\left(t-t_{0}\right)\right]},
$$

giving

$$
\begin{gathered}
\int d t\left(\dot{\phi}_{\max }^{d=1}\right)^{2}=n, \\
m^{2} \int d t\left(\phi_{\max }^{d=1}\right)^{2}=3 n .
\end{gathered}
$$

Since $\varphi(u)$, introduced in Eq. (28) and satisfying Eq. (29), is dimensionless (as is $u=m x$ ), and the corresponding $\phi_{\max }(x)$ has finite action, the quantity

$$
A \equiv \frac{1}{4} \int d^{d} u \varphi^{4}(u)
$$

is a finite, pure number greater than zero [8]. For the quantum-mechanical case mentioned above, $A=4 / 3$. For the cases $d>1, A$ is not explicitly known but, as noted, it must be a finite, positive, pure number. With the definition (38), Eq. (28) becomes

$$
\phi_{\max }(x)=m^{d / 2-1}\left(\frac{n}{A}\right)^{1 / 2} \varphi(m x)
$$

Since $\varphi(m x)$ satisfies the $n$-independent equation (29), we conclude that the field strength of $\phi_{\max }$ grows with the square root of the order $n$.

Equation (34), together with the definition (38) and the relation (39), allow us to write an expression for the action (26) at $\phi=\phi_{\max }$,

$$
S\left[\phi_{\max }\right]=2 n-n \ln \left[\frac{\lambda m^{d-4}}{A} n^{2}\right] .
$$

The value of $c_{n}[\phi(x)]$ at $\phi=\phi_{\max }$ then becomes, for large $n$,

$$
c_{n}\left[\phi_{\max }(x)\right] \approx \frac{1}{Z_{0}} \frac{(-1)^{n}}{2 \pi}(n-1) !\left(\frac{\lambda m^{d-4}}{A}\right)^{n} .
$$

With the change of variables

$$
\phi(x)=\phi_{\max }(x)+m^{d / 2-1} \phi_{q}(m x),
$$

the Gaussian approximation of $c_{n}[\phi]$ around $\phi_{\max }$ is 


$$
\begin{aligned}
c_{n}[\varphi(u)] \approx & \frac{1}{Z_{0}} \frac{(-1)^{n}}{2 \pi}(n-1) !\left(\frac{\lambda m^{d-4}}{A}\right)^{n} \\
& \times \exp \left(-\frac{1}{2} \int d^{d} u_{1} d^{d} u_{2} \phi_{q}\left(u_{1}\right)\left\{\left[-\nabla_{u_{1}}^{2}+1\right.\right.\right. \\
& \left.\left.\left.-3 \varphi^{2}\left(u_{1}\right)\right] \delta\left(u_{1}-u_{2}\right)\right\} \phi_{q}\left(u_{2}\right)\right) \\
& \times \exp \left(-\frac{1}{2} \int d^{d} u_{1} d^{d} u_{2} \phi_{q}\left(u_{1}\right)\right. \\
& \left.\times\left[(1 / A) \varphi^{3}\left(u_{1}\right) \varphi^{3}\left(u_{2}\right)\right] \phi_{q}\left(u_{2}\right)\right)
\end{aligned}
$$

where $u=m x$ and $\varphi(u)$, the solution of Eq. (29), is related to $\phi_{\max }$ through Eq. (39). This Gaussian approximation becomes exact in the limit $n \rightarrow \infty$.

The second derivative operator, which we call $D$, is, then,

$$
D=D_{\text {local }}+D_{\text {nonlocal }}
$$

with

$$
D_{\text {local }}=-\nabla^{2}+1-3 \varphi^{2}
$$

and

$$
D_{\text {nonlocal }}=\frac{1}{A}|v\rangle\langle v| \quad \text { with }\langle u \mid v\rangle=\varphi^{3}(u)
$$

and $A$ given in Eq. (38).

The operator $D_{\text {local }}$ is well known (see, for example, [8]). It has $d$ eigenvectors $\left|0_{\mu}\right\rangle$ with zero eigenvalues given by

$$
\left\langle u \mid 0_{\mu}\right\rangle=\frac{\partial}{\partial u^{\mu}} \varphi(u) .
$$

These vectors are also zero eigenvectors of $D$, as can be seen by noting that $|v\rangle$ is orthogonal to them:

$$
\left\langle v \mid 0_{\mu}\right\rangle=0
$$

They reflect the translation invariance of the action (26).

$D_{\text {local }}$ is also known to have one and only one negative eigenvector. $D$, on the contrary, is a positive semidefinite operator. We can prove this in a line-by-line analogy with the corresponding proof for $D_{\text {local }}$, which uses Sobolev inequalities and is given in Appendix 38 of Ref. [8],

$$
D \geqslant 0 \text {, }
$$

in the operator sense.

Projecting out the $d$-dimensional eigenspace of eigenvalue zero, the resulting operator, which we call $D^{\prime}$, is positive definite:

$$
D^{\prime}=D_{\text {local }}^{\prime}+D_{\text {nonlocal }}>0 \text {. }
$$

This equation explicitly states that the projection over the strictly positive eigenvectors modifies only $D_{\text {local }}$. The nonlocal part, as we saw, is a projector orthogonal to the zero modes and is therefore not modified under that operation.
Equations (50) and (51) suggest that the operator $D$, with the corresponding renormalization for $d>1$, generates a well-defined Gaussian measure in a finite volume (remember $d<4$ ). In fact, the determinant of $D_{\text {local }}^{\prime}$ has been calculated many times in the past [8], and a generalization of a quantum-mechanical argument of Ref. [18] indicates that this is all we need to compute the determinant of $D^{\prime}$. The argument goes as follows:

$$
\begin{aligned}
\operatorname{Det}\left[D^{\prime}\right] & =\operatorname{Det}\left[D_{\text {local }}^{\prime}+\frac{1}{A}|v\rangle\langle v|\right] \\
& =\operatorname{Det}\left[D_{\text {local }}^{\prime}\right]\left(1+\frac{1}{A}\left\langle v\left|D_{\text {local }}^{\prime-1}\right| v\right\rangle\right) .
\end{aligned}
$$

Since $\varphi(u)$ is orthogonal to $\partial_{\mu} \varphi(u)$ (the zero modes of $D$ and $\left.D_{\text {local }}\right)$,

$$
D_{\text {local }}^{\prime} \varphi=D_{\text {local }} \varphi=-2 \varphi^{3}
$$

The last equality follows from the definition of $D_{\text {local }}$ in Eq. (46) and Eq. (29) satisfied by $\varphi$. Inverting $D_{\text {local }}^{\prime}$ and remembering the definition of $|v\rangle$ and $A$ in Eqs. (47) and (38), we obtain

$$
\left\langle v\left|D_{\text {local }}^{\prime-1}\right| v\right\rangle=-2 A
$$

Replacing this result in Eq. (52), we arrive at the result

$$
\operatorname{Det}\left[D^{\prime}\right]=-\operatorname{Det}\left[D_{\text {local }}^{\prime}\right]
$$

As already mentioned, $D_{\text {local }}^{\prime}$ has one and only one negative eigenvector; consequently its determinant is negative. Equation (55) indicates then that $\operatorname{Det}\left[D^{\prime}\right]$ is positive, as it should be according to Eq. (51). The effect of the nonlocal part is to change the sign of the determinant of the local part.

The preceding equations allow us to integrate the Gaussian approximation of $c_{n}[\varphi(u)]$ given in Eqs. (43) and (44). Using the method of collective coordinates to project out the zero modes, the Jacobian of the corresponding change of variables is, at leading order in $1 / n$,

$$
J=\prod_{\mu=1}^{d}\left[\int\left(\partial_{\mu} \phi_{\max }\right)^{2} d^{d} x\right]^{1 / 2},
$$

where no sum over $\mu$ is implied.

It can be shown that the solutions of Eq. (27) corresponding to minimal action are spherically symmetric [8]. Equation (56) can then be written as

$$
J=\left[\frac{1}{d} \int\left(\partial_{\mu} \phi_{\max }\right)^{2} d^{d} x\right]^{d / 2},
$$

where now a sum over $\mu$ from 1 to $d$ is implied. Using Eq. (32) we then find

$$
J=n^{d / 2} \text {. }
$$

With this expression, the functional integral of $c_{n}[\varphi(u)]$ can be written as 


$$
\begin{aligned}
\frac{1}{Z_{0}} \int[d \phi] c_{n}[\phi]= & \frac{(-1)^{n}}{2 \pi}(n-1) !\left(\frac{\lambda m^{d-4}}{A}\right)^{n} \\
& \times\left(\operatorname{Vol} m^{d}\right) n^{d / 2}\left(-\operatorname{Det}\left[\frac{D_{\text {local }}^{\prime}}{D_{0}}\right]\right)^{-1 / 2},
\end{aligned}
$$

where $D_{0} \equiv-\nabla^{2}+1$. The factors in the line (59) correspond to the value of $c_{n}[\phi]$ at $\phi_{\max }$ up to the normalization $1 / Z_{0}$ as can be seen in Eq. (41). The factor "Vol" arises after the integration over the flat coordinates corresponding to the center of $\phi_{\max }$. The $n^{d / 2}$ comes from the Jacobian of the change of variables as mentioned before. The factor $m^{d}$ arises after the rescaling of the fields that makes them dimensionless in both $c_{n}[\phi]$ and $Z_{0}$. This happens because there are $d$ more integration variables in $Z_{0}$ due to the integration over the collective coordinates in the numerator. Finally, the factor $\left(-\operatorname{Det}\left[D_{\text {local }}^{\prime}\right]\right)^{-1 / 2}$ is the result of the integration over the coordinates orthogonal to the zero modes of $D$, while $\left(\operatorname{Det}\left[D_{0}\right]\right)^{1 / 2}$ is the dimensionless normalization factor (the mass dimension of both the numerator and the denominator was already taken care of in the term $m^{d}$ ). The minus sign is due to the nonlocal part of $D$ that, as proved above, simply changes the sign of the determinant of the local part, making it positive.

Equations (59) and (60) should agree with the corresponding result from Ref. [18] in the case $d=1$, where $-\operatorname{Det}\left[D_{\text {local }}^{\prime} / D_{0}\right]=1 / 12[8,18]$ and $A=4 / 3$. We see that the results are identical provided we take into account the different normalization here and a factor of 2 that accounts for the undetermined sign of the solution of Eq. (27), allowing both positive and negative solutions that contribute equally to the functional integral.

For $d=2$ or 3, the formal expressions (59) and (60) need of course to be renormalized. All the arguments in this section remain valid for the theory with a Pauli-Villars regularization [8]. The action (2) becomes

$$
\begin{aligned}
S[\phi]= & \int d^{d} x\left[\frac{1}{2} \phi\left(-\nabla^{2}+\frac{\nabla^{4}}{\Lambda^{2}}+m^{2}\right) \phi+\frac{\lambda}{4} \phi^{4}\right. \\
& \left.+\frac{1}{2} \delta m^{2}(\Lambda) \phi^{2}\right] .
\end{aligned}
$$

The modification of the kinetic part of the action affects both Eq. (27) and the scaling arguments, but by an amount that decreases as $\Lambda^{-2}$ when the ultraviolet cutoff $\Lambda$ becomes large.

As shown in Ref. [8], although the counterterm increases with the cutoff, it is also proportional to at least one power of $\lambda$. Therefore if we take the small $\lambda$ limit before the large cutoff limit, we are justified in ignoring the counterterm in Eq. (27) and in the scaling arguments. On the other hand, it contributes to the results (59) and (60) an amount that exactly cancels the divergence in the $\operatorname{Det}\left[D_{\text {local }}^{\prime}\right]$, making the final expression finite as it should be.

In the large $n$ limit, where the Gaussian approximations (43) and (44) become exact, the expressions (59) and (60) give the large order behavior of the perturbative series of $Z$ (up to the factor of 2 mentioned above) without any assump- tion about the analytic structure in $\lambda$ [18]. A completely analogous procedure would give the large order behavior of any Green's function.

Equations (39), (41), (43), (44), (59), and (60) allow us to draw an accurate picture of the mechanism underlying the lack of domination (in the sense of Lebesgue's theorem) of the convergence of the sequence of perturbative integrands (14) towards Eq. (16), and consequently of the mechanism underlying the divergence of the perturbative series. In fact, this picture is very similar to the one described in the previous section for the simple integral example. This is perhaps not surprising given the similarity of their large order behavior.

In a finite volume, there is a region of finite measure in field space in which the perturbative approximation $f_{N}[\phi(x)]$ of Eq. (14) approximates the exact integrand (16) with an error smaller than a given prescribed number. This region grows with $N$, becoming the full field space in the $N \rightarrow \infty$ limit. As in the simple example, this is a consequence of Taylor's theorem applied to the (analytic) integrand (16).

The problem is that, for any finite $N$, outside that region the approximate integrand $f_{N}[\phi(x)]$ strongly deviates from the exact one. This can be seen by noting that the maxima of every term of $f_{N}$ grow factorially with the order. Therefore, for large enough $N$, the last term is far greater than the previous ones at its maxima. Furthermore, as shown above, the Gaussian approximation around that maxima (which becomes exact for $N \rightarrow \infty$ ) defines a measure that does not go to zero as $N \rightarrow \infty$ [in fact, it is independent of $N$, Eqs. (43) and (44)]. This means that for every finite $N$, there is a region of finite measure in field space (and this measure does not go to zero as $N \rightarrow \infty$ ) in which the deviation between the perturbative integrand and the exact one is of the order of the maxima of the last term of $f_{N}$, i.e., of the order of $(N-1)$ !. No integrable functional can therefore satisfy the property (12) of Lebesgue's theorem.

This is the mechanism that makes the sequence of perturbative integrands, although convergent to the exact one, nondominated in the sense of Lebesgue's theorem. It is therefore also the mechanism that makes the sequence of integrals (i.e., the perturbative series) divergent. In fact, as Eqs. (59) and (60) show, the famous factorial growth of the large order coefficients of the perturbative series is a consequence, after integration, of exactly this behavior.

\section{STEPS TOWARDS A CONVERGENT SERIES}

It was mentioned in the Introduction that the analysis of the divergence of perturbation theory presented in this paper would point directly towards the aspects of the perturbative series that need to be modified in order to generate a convergent series. This is the topic of the present section.

In the previous section we analyzed perturbation theory from the point of view of the dominated convergence theorem. We have detected the precise way in which the convergence of the sequence of perturbative integrands to the exact one takes place and the way this convergence fails to be dominated. We have learned that for any finite order $N$, the field space naturally divides into two regions. In the first one, which grows with the order, eventually becoming the full field space (in the $N \rightarrow \infty$ limit), the perturbative integrands 
very accurately approximate the exact one. In the other one, however, the deviation between the perturbative and exact integrands is so strong that the sequence of integrals diverges.

It is then clear that if we could somehow modify the integrands, order by order, in the region where they deviate from the exact one, while preserving them as they are in the other region, then, with a "proper" modification, such a modified sequence of integrands would converge in a dominated way. According to the dominated convergence theorem, their integrals would then converge to the exact integral, achieving the desired goal of a convergent modified perturbation theory.

Let $\Omega_{N}$ be the region of field space in which the $N$ th perturbative integrand approximates with a given prescribed error the exact integrand (16). The characteristic function $\mathrm{Ch}\left(\Omega_{N},\{\phi(x)\}\right)$ of that region is equal to 1 for field configurations belonging to it and 0 otherwise:

$$
\operatorname{Ch}\left(\Omega_{N},\{\phi(x)\}\right) \equiv \begin{cases}1 & \text { for }\{\phi(x)\} \in \Omega_{N}, \\ 0 & \text { for }\{\phi(x)\} \notin \Omega_{N} .\end{cases}
$$

One possible realization of the above strategy of modifying the integrands (14) in the "bad" region of field space is to make them zero there. We would have

$$
\begin{aligned}
f_{N}^{\prime}[\phi(x)]= & \frac{1}{Z_{0}} \sum_{n=0}^{N} \frac{(-1)^{n}}{n !} e^{-S_{0}}\left(\frac{\lambda}{4} \int d^{d} x \phi^{4}\right)^{n} \\
& \times \operatorname{Ch}\left(\Omega_{N},\{\phi(x)\}\right) .
\end{aligned}
$$

According to the analysis of the previous section, if we choose $\Omega_{N}$ appropriately, the sequence of $f_{N}^{\prime}[\phi(x)]$ will exhibit dominated convergence, and the corresponding interchange between sum and integral will now be allowed. A rigorous proof of this is left for a paper currently in preparation. For the purposes of the present argument, it is sufficient to rely on the analysis of the previous section to assume its validity. Also, in the next section we will analyze, along the lines of the general ideas of this paper, some resummation schemes for which rigorous proofs of convergence have recently been given [9-16]. As that analysis will show, these methods strongly rely on the general notions underlying Eq. (63). Their convergence supports, then, the validity of the dominated nature of the convergence of Eq. (63) towards Eq. (16).

An urgent issue, however, is the practical applicability of the above strategy. To implement it, we need a functional representation of the characteristic function (62) (or an approximation to it) that only involves Gaussian and polynomial functionals. In the same way in which a functional representation of the Dirac $\delta$ function allows us to perform functional integrals with constraints, the Faddeev-Popov quantization of gauge theories being the most famous example, a functional representation of the characteristic function (62) would allow us to functionally integrate only the desired region of functional space. Since, basically, the functionals we know how to integrate reduce to Gaussians multiplied by polynomials, the desired representation of the characteristic function should only involve those functionals. Conversely, if it only involves those functionals, all the so- phisticated machinery developed for perturbation theory (including all the perturbative renormalization methods) would automatically be applicable. With this in mind, consider the function

$$
W(M, u) \equiv e^{-M u} \sum_{j=0}^{M} \frac{(M u)^{j}}{j !},
$$

where $M$ is a positive integer. Note that $W(M, u)$ arises from $1=e^{-M u} e^{+M u}$ by expanding the second exponential up to order $M$. Here $W(M, u)$ has the following remarkable properties.

(1) $W(M, u) \rightarrow 1$ when $M \rightarrow \infty$ for $0<u<1$. The convergence is uniform, with the error going to zero as

$$
R(M, u) \leqslant e^{M[\ln u-(u-1)]} \frac{1}{\sqrt{2 \pi M}} \frac{u}{1-u+1 / M} .
$$

(2) $W(M, u) \rightarrow 0$ when $M \rightarrow \infty$ for $1<u$. The convergence is also uniform, with an error of the form

$$
W(M, u) \leqslant e^{M[\ln u-(u-1)]} .
$$

As we see, the exponent corresponds to the same function in both cases. For $u>0$, this function is always negative except at its maximum, at $u=1$, where it is 0 . Therefore the convergence is in both cases exponentially fast in $M$, with the exponent becoming more and more negative, for a fixed $M$, when $u$ differs more and more from 1 . The proof of properties (1) and (2) is in Appendix A.

If we replace $u$ by a positive definite quadratic form $\langle\phi|D| \phi\rangle / C_{N}$, then the insertion of Eq. (64) into the functional integral would effectively cut off the region of integration $\langle\phi|D| \phi\rangle>C_{N}$ :

$$
\begin{aligned}
Z_{N}^{\prime}[\phi(x)]= & \frac{1}{Z_{0}} \int[d \phi] \sum_{n=0}^{N} \frac{\left(-S_{\text {int }}\right)^{n}}{n !} \\
& \times e^{-S_{0}} \lim _{M \rightarrow \infty} W\left(M, \frac{\langle\phi|D| \phi\rangle}{C_{N}}\right) \\
= & \frac{1}{Z_{0}} \sum_{n=0}^{N} \frac{(-1)^{n}}{n !} \lim _{M \rightarrow \infty} \int[d \phi] \\
& \times e^{-S_{0}\left(S_{\text {int }}\right)^{n} W\left(M, \frac{\langle\phi|D| \phi\rangle}{C_{N}}\right) .}
\end{aligned}
$$

$C_{N}$ is a constant that changes with the order $N$ of the expansion in $\lambda$, increasing with $N$ but in such a way that in the region where $\langle\phi|D| \phi\rangle<C_{N}$, the difference between the perturbative and the exact integrands is smaller than a given prescribed error. Since the convergence of $W$ is uniform according to properties (1) and (2), with errors given in Eqs. (65) and (66), the corresponding interchange between the sum in Eq. (68) and the functional integral is justified. The fact that $u$ becomes a quadratic form implies that the resulting integrands are Gaussians multiplied by monomials. Therefore the familiar Feynman diagram techniques can be used to integrate them. It also implies that no new loops 
appear and the sum in $j$ from Eq. (64) becomes an algebraic problem. A typical functional integral to compute has the form

$$
\begin{gathered}
\int[d \phi] \exp \left(-\int d^{d} x\left[\frac{1}{2}\left(\partial_{\mu} \phi\right)^{2}+\frac{1}{2} m^{2} \phi^{2}+\left(\phi D \phi / C_{N}\right)\right]\right) \\
\times\left(\int d^{d} x \phi^{4}\right)^{n}\left(\int d^{d} x \phi D \phi\right)^{m}
\end{gathered}
$$

as can be seen by replacing the definition (64) into Eq. (68) with $u=\langle\phi|D| \phi\rangle / C_{N}$.

Note that at any given order in $\lambda$ it is not necessary in principle to go to infinity in $M$. That would amount to replacing the perturbative integrands by zero in the region $\langle\phi|D| \phi\rangle>C_{N}$, realizing the strategy mentioned before. But since the convergence in $W$ is uniform, a finite, large enough $M$ (depending on the order in the expansion in the coupling constant) would suffice to tame the behavior of the perturbative integrands and transform them into a dominated convergent sequence. In fact, as we will see, many methods of improvement of perturbation theory use effectively formula (64) without sending $M \rightarrow \infty$ for any given finite order in perturbation theory. In any case, as already mentioned, that limit is in principle computable, since it does not involve new loops. Work in this direction is in progress.

The convergence of the sequence (68) towards $Z(\lambda)$ may be thought, at first sight, to be in conflict with our wellestablished knowledge about the nonanalyticity of this function at $\lambda=0$. In fact, Eq. (68) seems to be a power series in $\lambda$ (the powers of $\lambda$ coming from the powers of $S_{\text {int }}$ ); therefore, if convergent, that power series would define a function of $\lambda$ analytic at $\lambda=0$. It must be recognized, however, that the validity of Lebesgue's dominated convergence theorem is completely independent of any analyticity consideration. Therefore, if its hypothesis is satisfied, its conclusions must be valid. This being said, the question of how to reconcile the convergence of Eq. (68) with the nonanalyticity of $Z(\lambda)$ deserves an answer. To begin with, even at finite order in $\lambda$, the function (68) is not necessarily analytic at $\lambda=0$ despite its analytic appearance. This is because the constant $C_{N}$ may have an implicit nonanalytic dependence on $\lambda$. In Appendix $\mathrm{B}$ this is actually the case in the context of a simple example to which the present ideas are applied. But the mechanism that ultimately introduces the proper nonanalyticity in $\lambda$ is the limit process $N \rightarrow \infty$. Given a nonanalytic function such as $Z(\lambda)$ one can always construct a sequence of analytic functions that converge to it. Satisfying the hypothesis of the dominated convergence theorem is a way of achieving that, avoiding all the complicated and model-dependent issues of nonanalyticity. Note that the validity of this hypothesis for a given sequence of integrands can be checked independently of any analyticity consideration.

In Appendix B we prove the convergence of the general strategy discussed here for the simple integral example analyzed in Sec. (II B). For that case, making $u=\left(x / x_{c, N}\right)^{2}$, the function $W(M, u)$ becomes in the limit the characteristic function of the interval $|x|<x_{c, N}$. We use this to explicitly compute the nonanalytic function $z(\lambda)$ [Eq. (6)], calculating only Gaussian integrals. We also show explicitly how a nonanalytic dependence of $x_{c, N}$ on $\lambda$ naturally arises just by

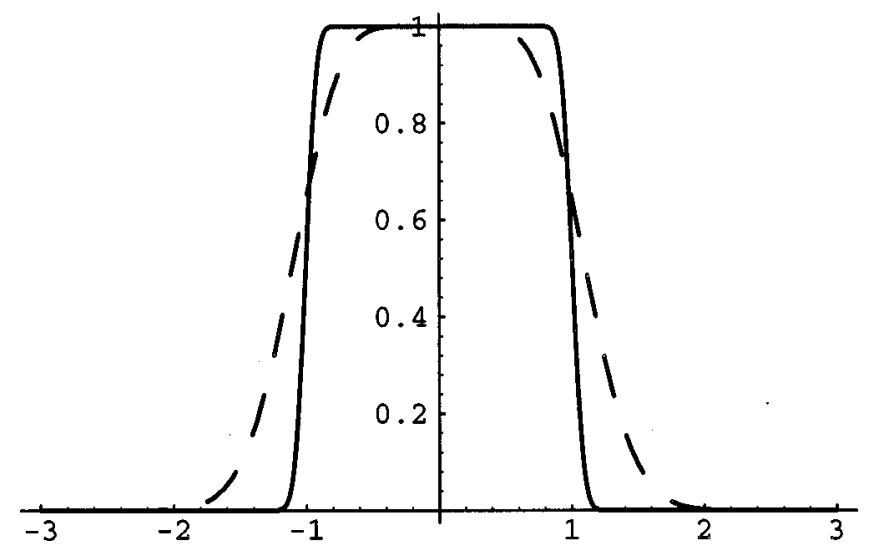

FIG. 3. Function $W\left(M, x, x_{c, N}\right)$ with $x_{c, N}=1$ for $M=3$ (dashed line) and $M=60$ (solid line). The convergence towards the characteristic function of the interval $|x|<x_{c, N}$ is apparent.

demanding the validity of Lebesgue's hypothesis and how the $N \rightarrow \infty$ limit process captures the full nonanalyticity of $z(\lambda)$. The same method also works for the "negative mass case," where the Borel resummation method fails. In Fig. 3, we can appreciate the convergence of $W$ towards the characteristic function of the interval $|x|<x_{c, N}$ for $x_{c, N}=1$ for two different values of $M$.

\section{IMPROVEMENT METHODS OF PERTURBATION THEORY}

The analysis of the mechanism of divergence of the perturbative series presented in this paper, together with the formula (64) and its properties, offers a large range of possibilities to construct a convergent series. In the previous section we have shown how that formula can be used to effectively cut off the region of field space where the strong deviation between perturbative and exact integrands takes place. But as we will see, this is only one possible way, among many, to use Eq. (64) to transform the sequence of perturbative integrands into a dominated one.

Another example of its possible use is the so-called "optimized $\delta$ expansion" $[19,20]$. In a series of papers [9$11,21]$, it was proved that such an expansion converges for the partition function of the anharmonic oscillator in finite Euclidean time. The problem of convergence in the infinite Euclidean time (or zero temperature) limit for the free energy or any connected Green's function is still under investigation, as well as its extension to quantum field theories $[10,12]$. The method was proved to generate a convergent series for the energy eigenvalues $[13,14]$, although such studies make heavy use of analyticity properties valid specifically in the models studied. In these works, it was realized that many methods of improvement of perturbation theory, such as the order-dependent mappings of Refs. $[22,23]$, possess the same general structure as the linear $\delta$ expansion. A considerable amount of work has been dedicated to investigating the virtues and limitations of the method and extensions of it $[11,15]$.

Although it is not appropriate to give a detailed analysis of these methods here, we would like to briefly indicate how they can be understood in terms of the ideas presented in this 
paper. In what follows, our analysis is restricted to $d=1$ (quantum mechanics) where rigorous results about the convergence of the methods considered here are available.

Let us consider the case of the anharmonic oscillator. Its action is given in Eq. (2) for $d=1$. The idea of the method is to replace it by an interpolating action

$$
\begin{aligned}
S_{\delta}= & \int d t\left[\frac{1}{2}\left(d_{t} \phi\right)^{2}+\frac{1}{2}\left(m^{2}+\frac{\lambda}{2 m} \alpha\right) \phi^{2}\right. \\
& \left.+\delta \frac{\lambda}{4}\left(\phi^{4}-\frac{\alpha}{m} \phi^{2}\right)\right] .
\end{aligned}
$$

Clearly, the dependence on the parameter $\alpha$ in $S_{\delta}$ is lost when $\delta=1$. For that value, the action (70) reduces to Eq. (2). However, if we expand up to a finite order in $\delta$ and then make $\delta=1$, the result still depends on $\alpha$. The idea is to tune $\alpha$, order by order in the expansion in $\delta$, so that the result is a convergent series. It was shown in the references mentioned above that the method works if $\alpha$ is tuned properly. For example, in Ref. [9], the asymptotic scaling $\alpha \simeq N^{2 / 3}$ was used to prove the convergence of the method for the partition function at finite Euclidean time.

It is interesting to note that, originally [9,21], $\alpha$ was tuned according to heuristic prescriptions such as the "principle of minimal sensitivity" [20] (at any given order in $\delta$, choose $\alpha$ so that the result is insensitive to small changes in it) or the criterion of "fastest apparent convergence" (the value of $\alpha$ at which the next order in $\delta$ vanishes). But later $[10,12]$, it was realized that the best strategy was simply to leave $\alpha$ undetermined, find an expression for the error (that obviously depends on $\alpha$ ), and then choose $\alpha$ so that the error goes to zero when the order in $\delta$ goes to infinity. It is clear that a structural understanding of the convergence of the method can help to construct the generalizations necessary to overcome the difficulties associated with the convergence in the infinite volume limit for connected Green's functions, as well as the extensions to general quantum field theories.

To understand the "optimized $\delta$ expansion" in terms of the ideas presented in this paper, let us expand the functional integral corresponding to the action (70) in powers of $\delta$ up to a finite order $N$, and make $\delta=1$ as the method indicates,

$$
\begin{aligned}
Z(m, \lambda, \alpha, N)= & \frac{1}{Z_{0}} \int[d \phi] \exp \left\{-\int d t\left[\frac{1}{2}\left(d_{t} \phi\right)^{2}\right.\right. \\
& \left.\left.+\frac{1}{2}\left(m^{2}+\frac{\lambda \alpha}{2 m}\right) \phi^{2}\right]\right\}\left[\sum_{n=0}^{N} \frac{(-1)^{n}}{n !}\right. \\
& \left.\times\left(\frac{\lambda}{4} \int \phi^{4}-\frac{\lambda \alpha}{4 m} \int \phi^{2}\right)^{n}\right]
\end{aligned}
$$

The general analysis of the mechanism of divergence of perturbation theory of Sec. II indicates that if the function (71) generates a convergent series with $\alpha$ scaling properly with $N$, then, barring miraculous coincidences, the corresponding integrands should converge in a dominated way (or, even better, uniformly) towards the exact integrand (14). We want to obtain a qualitative understanding of how this method achieves that.
Expanding the binomial and making some elementary changes of variables in the indices of summation, we obtain the expression

$$
\begin{aligned}
Z(m, \lambda, \alpha, N)= & \frac{1}{Z_{0}} \int[d \phi] \exp \left\{-\int d t\left[\frac{1}{2}\left(d_{t} \phi\right)^{2}\right.\right. \\
& \left.\left.+\frac{1}{2}\left(m^{2}+\frac{\lambda \alpha}{2 m}\right) \phi^{2}\right]\right\}\left\{\sum_{i=0}^{N} \frac{(-1)^{i}}{i !}\right. \\
& \left.\times\left(\frac{\lambda}{4} \int \phi^{4}\right)^{i}\left[\sum_{k=0}^{N-i} \frac{1}{k !}\left(\frac{\lambda \alpha}{4 m} \int \phi^{2}\right)^{k}\right]\right\} .
\end{aligned}
$$

This equation already shows some of the distinctive characteristics of the method. As we see, the $i$ th power of the interacting action in the expansion of $e^{-S_{\text {int }}}$ up to order $N$ is multiplied by

$$
\mathcal{W}(N-i) \equiv \exp \left(-(\lambda \alpha / 4 m) \int \phi^{2}\right)\left[\sum_{k=0}^{N-i} \frac{1}{k !}\left(\frac{\lambda \alpha}{4 m} \int \phi^{2}\right)^{k}\right] .
$$

Note that $\mathcal{W}(N)$ corresponds to the function $W(M, u)$ with $M=N$ ( $N$ is the order in the expansion of $e^{-S_{\text {int }}}$ ) and the variable $u$ replaced by the quadratic form $\left[(\lambda / 4 m) \int \phi^{2}\right] / C_{N}$, where $C_{N}=N / \alpha$. Taking, for example, $\alpha \simeq N^{2 / 3}$ as in Ref. [9] (where it was proved that with such a scaling the method generates a convergent series), we see that, according to the previous section, $\mathcal{W}(N)$ is an approximation of the $\theta$ function in the region of field space characterized by

$$
\frac{\lambda \alpha}{4 m} \int d x \phi^{2} \leqslant N^{1 / 3}
$$

Equation (72), however, shows that the mechanism used to achieve dominated convergence cannot be reduced to a simple insertion of the function $W(M, u)$ with $M=N$ and $u=\left[(\lambda / 4 m) \int \phi^{2}\right] / C_{N}$. That would be the case if all the powers of the expansion of $e^{-S_{\text {int }}}$ up to order $N$ were multiplied by $\mathcal{W}(N)$. But Eq. (72) shows that the $i$ th power of the interacting action is in fact multiplied by $\mathcal{W}(N-i)$.

At this point it is convenient to pause for a moment in our study of the "optimized $\delta$ expansion" to give some useful definitions.

Let us call passive mechanisms (to achieve dominated or uniform convergence of a sequence of integrands to the exact one) those that can be reduced to the product of the $N$ th perturbative integrand and the characteristic function of a region $\Omega_{N}$ of field space for some sequence $\left\{\Omega_{N}\right\}$.

Passive methods use only information that is already available in the perturbative integrands; they just get rid of the "noise" inherent to perturbation theory. Because of that, in addition to defining a convergent series, they can also be very useful for studying perturbation theory itself. The function $W(N, u)$, with $u$ replaced by a properly selected quadratic operator, was specially designed to make passive methods practical. In a sense, Sec. III is a discussion of passive methods. 
Active mechanisms are those that are not passive, as defined above.

What kind of mechanism is the one underlying the "optimized $\delta$ expansion' method?

A trivial generalization of the proof, in the previous section, of the convergence of $W(M, u)$ towards the $\theta$ function for $u>0$ shows that the function

$$
\bar{W}(M, u, i) \equiv e^{-M u} \sum_{n=0}^{M-i} \frac{(M u)^{i}}{i !}
$$

also converges towards the $\theta$ function for $u>0$ in the limit

$$
M \rightarrow \infty, \quad i \text { fixed. }
$$

In this sense, the "optimized $\delta$ expansion" method does have passive aspects. As Eqs. (72) and (73) show, it amounts to multiplying the $i$ th power of the expansion up to order $N$ of $e^{S_{\text {int }}}$ by $\bar{W}$ with $u=\left[(\lambda / 4 m) \int \phi^{2}\right] / C_{N}$ and $C_{N}=N / \alpha$. Since this function converges to the characteristic function of the region characterized by Eq. (74), this means that the first $i$ terms of the expansion up to order $N$ of $e^{S_{\text {int }}}$ are effectively multiplied by the same function (an approximate characteristic function) for $i \ll N$. Therefore, the first $i$ terms, with $i \ll N$, use only the information available in the perturbative series to converge to the exact integrand.

What about the other terms, i.e., the ones characterized by $i \lesssim N$ ? Surprisingly, these terms produce a convergence of the corresponding integrands towards the exact one that is faster than possible with only passive components.

It is not the place here to study this aspect in detail, and so let us simply show this "faster than passive" convergence for the simple integral example.

Applied to the "massless" version of the integral (6), the optimized $\delta$ expansion method was proved to generate a rapidly convergent sequence in Ref. [21]. That is, the sequence given by

$$
I_{N} \equiv \sum_{n=0}^{N} \frac{(-1)^{n}}{n !} \int_{-\infty}^{\infty} d x e^{-\alpha(N) x^{2}}\left(\frac{\lambda}{4} x^{4}-\alpha(N) x^{2}\right)^{n}
$$

was proved to converge to

$$
I \equiv \int_{-\infty}^{\infty} d x e^{-\lambda x^{4} / 4}
$$

when $\alpha(N) \simeq \sqrt{N}$ with an error that goes to zero at the very fast rate of $R_{N}<C N^{1 / 4} e^{-0.663 N}$ when $N \rightarrow \infty$. $C$ is a numerical constant.

We are interested in understanding whether the corresponding convergence of the integrands is faster than passive. For our qualitative purposes, it is enough to observe, in Fig. 4, the convergence towards the exact integrand

$$
I_{\mathrm{exa}}(x)=e^{-(\lambda / 4) x^{4}}
$$

of both the perturbative integrand

$$
I_{\text {pert }}=\sum_{n=0}^{N} \frac{\left(-\lambda x^{4} / 4\right)^{n}}{n !}
$$

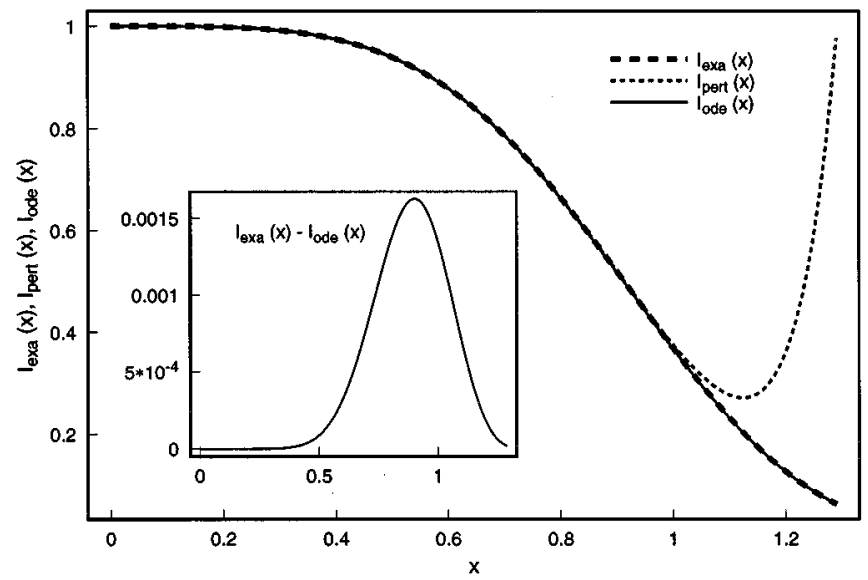

FIG. 4. In the main plot, the superiority of the convergence of the fourth order optimized $\delta$ expansion ("ode") with respect to the same order perturbative approximation is evident. In the subgraph, the difference between the "ode" and the exact integrand is plotted. Note the difference in the scales of the $y$ axis of the main and subgraph.

and the optimized $\delta$ expansion integrand

$$
I_{\text {ode }}=\sum_{n=0}^{N} \frac{(-1)^{n}}{n !} e^{-\alpha(N) x^{2}}\left(\frac{\lambda}{4} x^{4}-\alpha(N) x^{2}\right)^{n},
$$

with $\alpha(N) \simeq \sqrt{N}$, for $N=4$.

We can see how accurate the convergence of $I_{\text {ode }}(x)$ is, even at this low order. In particular, when the perturbative integrand begins to diverge, $I_{\text {ode }}(x)$ continues to approximate the exact integrand remarkably well. In the inset, we can appreciate the difference between $I_{\text {exa }}(x)$ and $I_{\text {ode }}(x)$. Note the difference in the $y$ axis scale of the main graph and the inset.

It is then clear that the optimized $\delta$ expansion method, with its subtle combination of passive and active components, manages to generate a sequence of integrands that (uniformly) converges towards the exact one at a rate that far exceeds the possibilities within a purely passive method.

From this qualitative discussion of the optimized $\delta$ expansion method we can deduce two general lessons: (1) Any method of improvement of the perturbative series in a given quantum theory, where a functional integral representation of the quantity under study exists, must rely, at the level of the integrands, on an improvement over the pointwise convergence of the Taylor series in the coupling constants of $e^{-S}$; (2) the problem of finding a convergent series reduces to the problem of finding a dominated convergent sequence of integrands towards $e^{-S}$. This second simple statement not only provides a guide to the construction of convergent schemes, but also emphasizes the fact that, in principle, a dominated convergent sequence of integrands does not have to have any relation whatsoever to the corresponding Taylor expansion. In order to be able to use the usual techniques of quantum field theory, it is reasonable to restrict the search for a convergent scheme to a sequence of integrands of the general form 


$$
f_{N}=e^{-S_{0}} \sum_{n=0}^{N} a_{n} S_{\text {Int }}^{n} f_{n}\left(\left\langle\phi\left|D_{n}\right| \phi\right\rangle\right),
$$

where the functional $f_{n}$ of the quadratic form $\left\langle\phi\left|D_{n}\right| \phi\right\rangle$ should take care of the nondominated convergence that is bound to appear with only powers of the interacting action. The function $W$ of Sec. III, with its possible generalizations, is an ideal candidate for this purpose. But the selection of the coefficients $a_{n}$ amounts to a pure problem in optimization of the convergence of the integrands - no a priori connection with any Taylor series is necessary.

\section{CONCLUSIONS}

In this paper we have exposed the mechanism, at the level of the integrands, that makes the perturbative expansion of a functional integral divergent. We have seen in detail how the sequence of integrands violates the domination hypothesis of Lebesgue's dominated convergence theorem. That theorem, as is well known, establishes the conditions under which one is allowed to interchange an integration and a limit, in particular the interchange that takes place in the generation of perturbation series.

It was shown that at any finite order in perturbation theory, the field space divides into two regions. In one region, whose measure grows with the order, the perturbative integrands very accurately approximate the exact integrand. In the other region, however, a strong deviation takes place. It was shown that the behavior in this second region violates the hypothesis of Lebesgue's theorem and, consequently, generates the divergence of perturbation theory. The famous factorial growth of the large order coefficients of the perturbative series was shown to be an effect, after integration, of the very mechanism that violates the hypothesis of the theorem.

All of the above was done explicitly without relying on the particular analytic properties of the models studied. It is therefore natural to assume that similar mechanisms of the violation of Lebesgue's hypothesis are present in any other quantum field theory, although for just renormalizable theories other mechanisms are responsible for renormalons. Studies in this direction are in progress.

The mechanism of divergence presented here points towards a simple way to achieve a convergent series: Integrate only in the "good" region of field space. Since this region grows with the order, becoming in the limit the whole field space, integrating in a correspondingly increasing region we would obtain a convergent series. A step towards a practical implementation of this program was made with the construction of the function $W$, Eq. (64). This function allows us to introduce a Gaussian representation of the characteristic function of regions of field space, in much the same way that the imposition of constraints in the functional integral was allowed by a functional representation of the Dirac $\delta$ function. A rigorous proof of the convergence of this practical implementation of the above-mentioned strategy is in progress. In Appendix B it was applied to a simple integral example.

Finally, a qualitative analysis of the optimized $\delta$ expansion method of improvement of perturbation theory in terms of the ideas of this paper was presented. Some general prop- erties of improvement methods, useful to generate new schemes, as well as to understand and improve old ones, have been established.

\section{ACKNOWLEDGMENTS}

S.P. was supported in part by U.S. Department of Energy Grant No. DE-FG 02-91ER40685. He would like to thank A. Duncan for numerous very useful discussions, and also A. Das, L. Orr, and S. Rajeev.

\section{APPENDIX A}

In this appendix we will prove the two properties of formula (64).

Because for $u>0$ all the terms of the sum defining $W(M, u)$ are positive, we have trivially $W(M, u)>0$. On the other hand, since in the Taylor expansion of $e^{M u}$ all the terms are positive, we have $\sum_{n=0}^{M}(M u)^{n} / n ! \leqslant e^{M u}$. Therefore $W(M, u) \leqslant 1$. So for every $M$ and positive or zero $u$ we have

$$
0 \leqslant W(M, u) \leqslant 1
$$

Consider first the case $0<u<1$ :

$$
1-W(M, u)=e^{-M u} \sum_{n=M+1}^{\infty} \frac{(M u)^{n}}{n !} \equiv R(M, u) .
$$

We will prove that $R(M, u) \rightarrow 0$ when $M \rightarrow \infty$.

Changing variables to $j=n-M$, we get

$$
\begin{aligned}
R(M, u) & =e^{-M u} \frac{(M u)^{M}}{M !} \sum_{j=1}^{\infty}(M u)^{j} \frac{M !}{(j+M) !} \\
& \leqslant e^{-M u} \frac{(M u)^{M}}{M !} \sum_{j=1}^{\infty} \frac{(M u)^{j}}{(M+1)^{j}} \\
& \leqslant e^{-M u} \frac{(M u)^{M}}{M !} \frac{u}{1-u+1 / M} .
\end{aligned}
$$

But $M^{M} / M ! \rightarrow e^{M} / \sqrt{2 \pi M}$ for large $M$, and so

$$
R(M, u) \leqslant e^{M[\ln u-(u-1)]} \frac{1}{\sqrt{2 \pi M}} \frac{u}{1-u+1 / M} .
$$

The exponent is negative in the region $0<u<1$ since both $\ln u$ and $(u-1)$ are negative there and $|\ln u|>|u-1|$. Therefore

$$
R(M, u) \rightarrow 0 \quad \text { when } M \rightarrow \infty
$$

in the region $0<u<1$ and property (1) is proved with an exponentially fast convergence.

In the region $u>1$, we have

$$
\begin{aligned}
W(M, u) & =e^{-M u} \sum_{n=0}^{M} \frac{(M u)^{n}}{n !} \leqslant e^{-M u} u^{M} \sum_{n=0}^{M} \frac{M^{n}}{n !} \\
& \leqslant e^{M[\ln u-(u-1)]} .
\end{aligned}
$$


The first inequality is valid because $u>1$ and the second because $e^{M}>\sum_{n=0}^{M} M^{n} / n !$. The exponent is again negative. For $u>1$, both $\ln u$ and $(u-1)$ are positive, but now $|\ln u|<|u-1|$. So property (2) is also valid with an exponentially fast convergence.

For $u=1$ all we know is that $W$ is bounded by Eq. (A1). That is all we need. Numerics suggest $W(M, 1) \rightarrow 1 / 2$ when $M \rightarrow \infty$.

This finishes our proof.

\section{APPENDIX B}

In this appendix we apply the strategy discussed in Sec. III to generate a series convergent to the function $z(\lambda)$ [Eq. (6)]. This is done using the function $W$ of Eq. (64) and computing exclusively Gaussian integrals; therefore, we restrict ourselves to using only those techniques that are also available in quantum field theory.

As mentioned in Sec. III, the simplest possible modification of the perturbative integrand (15) that would transform the corresponding sequence into a dominated one amounts to keeping them as they are for $|x|<x_{c, N}$ and replacing them by zero for $|x|>x_{c, N}$. That is,

$$
f_{N}^{\prime}= \begin{cases}\pi^{-1 / 2} \sum_{n=0}^{N} \frac{(-1)^{n}}{n !}\left(\frac{\lambda}{4} x^{4}\right)^{n} e^{-x^{2}} & \text { for }|x|<x_{c, N}, \\ 0 & \text { for }|x|>x_{c, N} .\end{cases}
$$

In fact, choosing $x_{c, N}$ so as to properly avoid the region in which the deviation takes place, the sequence of $f_{N}^{\prime}$ converges uniformly towards the exact integrand (17) as we will show shortly. Consequently, the corresponding sequence of integrals

$$
\begin{aligned}
\int_{-\infty}^{\infty} d x f_{N}^{\prime} & =\pi^{-1 / 2} \int_{-x_{c, N}}^{x_{c, N}} d x \sum_{n=0}^{N} \frac{(-1)^{n}}{n !}\left(\frac{\lambda}{4} x^{4}\right)^{n} e^{-x^{2}} \\
& =\pi^{-1 / 2} \sum_{n=0}^{N} \int_{-x_{c, N}}^{x_{c, N}} d x \frac{(-1)^{n}}{n !}\left(\frac{\lambda}{4} x^{4}\right)^{n} e^{-x^{2}}
\end{aligned}
$$

will converge to the desired integral

$$
z(\lambda)=\pi^{-1 / 2} \int_{-\infty}^{\infty} d x e^{-\left[x^{2}+(\lambda / 4) x^{4}\right]} .
$$

In Eq. (B2) the change in the limits of integration from $\pm \infty$ to $\pm x_{c, N}$ is just due to the definition of $f_{N}^{\prime}$ in Eq. (B1). The interchange between sum and integral in Eq. (B3) is now allowed because in the region $\left[-x_{c, N}, x_{c, N}\right]$ we have uniform convergence (this is a stronger condition than dominated convergence). The resulting integrals are not Gaussian due to the finite limits of integration. We will show how they can be calculated using only Gaussian integrals.

A trivial way to achieve convergence of the sequence of integrals of the $f_{N}^{\prime}$ of Eq. (B1) towards Eq. (B4) amounts to keeping $x_{c, N}$ equal to a finite constant ' $a$ ', independent of $N$, while taking the limit $N \rightarrow \infty$. In this limit, Eq. (B3) becomes identical to $\pi^{-1 / 2} \int_{-a}^{a} d x e^{-\left[x^{2}+(\lambda / 4) x^{4}\right]}$, since for finite $a$ the Taylor series of the integrands converges uniformly. Therefore, as already said, the interchange between sum and integral is legal. Finally, taking the limit $a \rightarrow \infty$, we would obtain the desired convergence towards $z(\lambda)$.

However, better use can be made of the information available in $f_{N}^{\prime}$ for finite $N$. For example, for every finite $N$, we can choose $x_{c, N}$ so that

$$
\left|f_{N}^{\prime}(x)-f(x)\right| \leqslant \frac{\epsilon_{T, N}}{2 x_{c, N}} \quad \text { for }|x|<x_{c, N},
$$

with $\epsilon_{T, N}$ going to zero as $N \rightarrow \infty$. Then, since we have

$$
\left|f_{N}^{\prime}(x)-f(x)\right| \leqslant e^{-\left[x_{c, N}^{2}+(\lambda / 4) x_{c, N}^{4}\right]} \equiv \frac{\epsilon_{c, N}}{2} \quad \text { for }|x|>x_{c, N},
$$

the $f_{N}^{\prime}(x)$ will uniformly converge towards the exact integrand $f(x)$ if Eq. (B5) is consistent with $x_{c, N} \rightarrow \infty$ when $N \rightarrow \infty$. Indeed, if this happens, we would have

$$
\left|\int_{-\infty}^{\infty}\left[f(x)-f_{N}(x)\right] d x\right| \leqslant \epsilon_{T, N}+\epsilon_{c, N} \rightarrow 0 \quad \text { when } N \rightarrow \infty .
$$

The term $\epsilon_{T, N}$ comes trivially from Eq. (B5), while $\epsilon_{c, N}$ comes from Eq. (B6) and the inequality

$$
\int_{x_{c, N}}^{\infty} e^{-\left[x^{2}+(\lambda / 4) x^{4}\right]} d x \leqslant e^{-\left[x_{c, N}^{2}+(\lambda / 4) x_{c, N}^{4}\right]}=\epsilon_{c, N},
$$

valid for $x_{c, N}>1$.

Applying Taylor's theorem to the function $e^{-\lambda x^{4} / 4}$ one can easily show that the condition (B5) is satisfied if

$$
x_{c, N}=\left[(N+1) ! \frac{\epsilon_{T, N}}{2}\left(\frac{4}{\lambda}\right)^{(N+1)}\right]^{1 /[4(N+5 / 4)]} .
$$

Note that the nonanalytic dependence of $x_{c, N}$ on $\lambda$ arises automatically from the imposition of Eq. (B5) to satisfy the hypothesis of Lebesgue's theorem.

Remember that the only condition on $\epsilon_{T, N}$ (in order to achieve convergence of the sequence of integrals) is to go to zero when $N \rightarrow \infty$ consistently with $x_{c, N} \rightarrow \infty$ in that limit. Choosing, for example,

$$
\epsilon_{T, N}=e^{-4 N^{1 / 4}},
$$

we obtain, asymptotically,

$$
x_{c, N} \rightarrow(4 N / e \lambda)^{1 / 4} .
$$

This implies [through Eq. (B6)]

$$
\boldsymbol{\epsilon}_{c, N} \rightarrow e^{-(4 N / e \lambda)^{1 / 2}-N / e} .
$$

Equations (B10) and (B12) show the exponential rate at which the convergence of the sequence of integrals takes place.

Clearly the form (B10) for $\epsilon_{T, N}$ is not unique, nor even the most efficient one, but enough to achieve convergence. 
TABLE I. Integration over the small field configurations only produces a convergent series. In the last column the improvement over the perturbative values can be appreciated.

\begin{tabular}{cccc}
\hline \hline Order & $\begin{array}{c}\text { Exact value } \\
(\lambda=4 / 10)\end{array}$ & Convergent series & Perturbative series \\
\hline 2 & 0.837043 & 0.803160 & 0.848839 \\
4 & 0.837043 & 0.830264 & 0.854087 \\
6 & 0.837043 & 0.835516 & 0.901897 \\
8 & 0.837043 & 0.836667 & 1.316407 \\
20 & 0.837043 & 0.837044 & $2.33755 \times 10^{8}$ \\
\hline \hline
\end{tabular}

In Table I one can appreciate the numerical convergence for $\lambda=4 / 10$.

Up to now we have proved that the general strategy of Sec. III does, in fact, generate a convergent sequence towards $z(\lambda)$. However, the resulting integrals in Eq. (B3) are not Gaussians, making the applicability of the method in quantum field theory dubious, to say the least. We will show now that the integrals of Eq. (B3) can be computed, using Eq. (64) with $u=\left(x / x_{c, N}\right)^{2}$, calculating only Gaussian integrals. The steps involved are

$$
\begin{aligned}
\int_{-x_{c, N}}^{x_{c, N}} x^{r} e^{-x^{2}} d x= & \int_{-\infty}^{\infty} x^{r} e^{-x^{2}} \lim _{M \rightarrow \infty} W\left(M, x, x_{c, N}\right) d x \\
= & \lim _{M \rightarrow \infty} \int_{-\infty}^{\infty} x^{r} e^{-x^{2}} W\left(M, x, x_{c, N}\right) d x \\
= & \lim _{M \rightarrow \infty} \sum_{n=0}^{M} \frac{1}{n !}\left(\frac{M}{x_{c, N}^{2}}\right)^{n} \int_{-\infty}^{\infty} \\
& \times e^{-\left(1+M / x_{c, N}^{2}\right) x^{2}} x^{2 n+r} d x .
\end{aligned}
$$

The two properties of $W$ validate both equalities (B13) and (because of the uniformity of the convergence in $W$ ) (B14). In the last line, Eq. (B15), we just make explicit the meaning of Eq. (B14). So it is clear that these two properties are enough to prove the validity of Eq. (B15), where only Gaussian integrals are present. But it is a good exercise to find a direct proof of it in the case at hand, where everything can be computed exactly. We do this next.

For $r$ odd the integrals vanish, and so let us consider the case where $r$ is even, that is, $r=2 t$, for any integer $t$.

On the one hand, we have

$$
\int_{-x_{c, N}}^{x_{c, N}} x^{2 t} e^{-x^{2}} d x=\left(x_{c, N}\right)^{2 t+1} \sum_{k=0}^{\infty} \frac{(-1)^{k}}{k !} \frac{\left(x_{c, N}\right)^{2 k}}{(k+t+1 / 2)}
$$

where the necessary interchange between sum and integral to arrive at the result is allowed due to the uniform convergence of the Taylor series of $e^{-x^{2}}$ in the finite segment $\left[-x_{c, N}, x_{c, N}\right]$.

On the other hand,

$$
\begin{aligned}
& \lim _{M \rightarrow \infty} \sum_{n=0}^{M} \frac{1}{n !}\left(\frac{M}{x_{c, N}^{2}}\right)^{n} \int_{-\infty}^{\infty} e^{-\left(1+M / x_{c, N}^{2}\right) x^{2} x^{2(n+t)} d x} \\
&=\lim _{M \rightarrow \infty} \sum_{n=0}^{M} \frac{1}{n !} \Gamma(n+t+1 / 2) \\
& \times\left(\frac{x_{c, N}^{2}}{M}\right)^{t+1 / 2}\left(1+\frac{x_{c, N}^{2}}{M}\right)^{-(n+t+1 / 2)} \\
&= \lim _{M \rightarrow \infty} \sum_{n=0}^{M} \frac{1}{n !}\left(\frac{x_{c, N}^{2}}{M}\right)^{t+1 / 2} \sum_{k=0}^{\infty} \frac{(-1)^{k}}{k !} \\
& \times \Gamma(n+t+k+1 / 2)\left(\frac{x_{c, N}^{2}}{M}\right)^{k} \\
&=\left(x_{c, N}\right)^{2 t+1} \sum_{k=0}^{\infty} \frac{(-1)^{k}}{k !} \frac{\left(x_{c, N}\right)^{2 k}}{(k+t+1 / 2)} \\
& \times\left[\lim _{M \rightarrow \infty} \frac{(k+t+1 / 2)}{M^{(k+t+1 / 2)}} \sum_{n=0}^{M} \frac{\Gamma(n+t+k+1 / 2)}{n !}\right] .
\end{aligned}
$$

In Eq. (B18) we have used the equation

$$
\int_{-\infty}^{\infty} x^{2 n} e^{-p x^{2}} d x=\frac{\Gamma(n+1 / 2)}{p^{n+1 / 2}},
$$

in Eq. (B19) we have expanded the last term of Eq. (B18) in powers of $x_{c, N}^{2} / M$ and carried out some cancellations, and finally in Eq. (B20) we have interchanged the $M \rightarrow \infty$ limit with the infinite sum in $k$.

Comparing Eqs. (B16) and (B20), we see that the validity of Eq. (B15) depends on the validity of the equation

$$
\begin{aligned}
& \lim _{M \rightarrow \infty} \frac{(k+t+1 / 2)}{M^{(k+t+1 / 2)}} \sum_{n=0}^{M} \frac{\Gamma(n+k+t+1 / 2)}{n !} \\
& \quad=1 \quad \forall \text { integers } k, t>0 .
\end{aligned}
$$

That this identity holds for every integer $t$ and $k$ can be seen by considering the following analytic function of the complex variable $z$ :

$$
O(z) \equiv \lim _{M \rightarrow \infty} \frac{(1 / z)}{M^{(1 / z)}} \sum_{n=0}^{M} \frac{\Gamma(n+1 / z)}{\Gamma(n+1) !} .
$$

If the identities (B22) hold, this function must be identically 1 , since for $1 / z_{j}=j+1 / 2$ with $j$ integer it reduces to them, and for ever increasing $j$, we obtain a sequence accumulating at $z=0$ on which the function should be 1 .

Conversely we will prove that $O(z)$ is indeed identically 1 as an analytic function of $z$, proving in consequence the identities (B22) for arbitrary $t$ and $k$. Consider the sequence $1 / z_{j}=j+1$ for $j$ integer. This sequence also accumulates at $z=0$, and for all its points we have 


$$
\begin{aligned}
& O(1 /(j+1))=\lim _{M \rightarrow \infty} \frac{(j+1)}{M^{(j+1)}} \sum_{n=0}^{M} \frac{\Gamma(n+j+1)}{\Gamma(n+1)} \\
& =\lim _{M \rightarrow \infty} \frac{(j+1)}{M^{(j+1)}} \sum_{n=0}^{M} \Pi_{i=1}^{j}(i+n) \\
& =\lim _{M \rightarrow \infty} \frac{(j+1)}{M^{(j+1)}}\left[\sum_{n=0}^{M} n^{j}+\mathcal{O}\left(n^{j-1}\right)\right] \\
& =\lim _{M \rightarrow \infty} \frac{(j+1)}{M^{(j+1)}}\left[\frac{M^{(j+1)}}{(j+1)}+\mathcal{O}\left(M^{j}\right)\right] \stackrel{M \rightarrow \infty}{\rightarrow} 1 .
\end{aligned}
$$

Therefore $O(z)=1$ for all $z$. This finishes the direct proof of Eq. (B15).
As was mentioned before, Eq. (B9), derived independently of any analyticity consideration, and only with the purpose of satisfying the hypothesis of Lebesgue's theorem, introduces a nonanalyticity in the sequence of integrals of $f_{N}^{\prime}$ even for finite $N$. But even for the case where $x_{c, N}$ is fixed to a constant $a$, discussed before, in which the limit $N \rightarrow \infty$ is taken first, and then $a$ is sent to infinity, and therefore the sequence is made out of truly analytic functions, the convergence towards $z(\lambda)$ is perfectly compatible with analyticity considerations. The functions $\pi^{-1 / 2} \int_{-a}^{a} d x e^{-\left[x^{2}+(\lambda / 4) x^{4}\right]}$ (the result of the $N \rightarrow \infty$ limit) are clearly analytic in $\lambda$. But they converge to (in fact they define) the nonanalytic function $z(\lambda)$ when $a \rightarrow \infty$. The limit of an infinite sequence of analytic functions does not have to be analytic.

Another important issue is that the same method also works for the "negative mass case," where the Borel resummation method fails. Indeed, from the discussion of this appendix it must be obvious that, with a proper scaling of $x_{c, N}$, the $f_{N}^{\prime}$ 's with a negative quadratic part of the exponent also converge uniformly towards $e^{\left[x^{2}-(\lambda / 4) x^{4}\right]}$ for $x$ in $\left[-x_{c, N}, x_{c, N}\right]$. Therefore, the sequence of integrals is also convergent.
[1] F. J. Dyson, Phys. Rev. 85, 631 (1951).

[2] Large-Order Behaviour of Perturbation Theory, edited by J. C. Le Guillou and J. Zinn-Justin (North-Holland, Amsterdam, 1990).

[3] C. M. Bender and T. T. Wu, Phys. Rev. D 7, 1620 (1973).

[4] C. M. Bender and T. T. Wu, Phys. Rev. 148, 1231 (1969).

[5] B. Simon, in Cargese Lectures in Physics, edited by D. Bessis (Gordon and Beach, New York, 1972), Vol. 5; J. J. Loeffel et al., Phys. Lett. 30B, 1656 (1968); S. Graffi et al., ibid. 32B, 631 (1970); B. Simon, Functional Integration and Quantum Physics (Academic Press, New York, 1979).

[6] See, for example, G. 't Hooft, in Erice Lectures 1977, edited by A. Zichichi (Plenum, New York, 1979).

[7] G. Hardy, Divergent Series (Oxford University Press, New York, 1949); E. Borel, Lecons sur les series divergent (1922).

[8] J. Zinn-Justin, Quantum Field Theory and Critical Phenomena, 2nd ed. (Clarendon, Oxford, 1993).

[9] A. Duncan and H. F. Jones, Phys. Rev. D 47, 2560 (1993).

[10] C. M. Bender, A. Duncan, and H. F. Jones, Phys. Rev. D 49, 4219 (1994).

[11] H. Kleinert, Phys. Lett. A 173, 332 (1993); J. Jaenicke and H. Kleinert, ibid. 176, 409 (1993); H. Kleinert and H. Meyer, ibid. 184, 319 (1994); W. Janke and H. Kleinert, Phys. Lett. A 199, 287 (1995); W. Janke and H. Kleinert, quant-ph/9502019.

[12] C. Arvanitis, H. F. Jones, and C. S. Parker, Phys. Rev. D 52, 3704 (1995).

[13] R. Guida, K. Konishi, and H. Suzuki, Ann. Phys. (N.Y.) 241, 152 (1995).

[14] R. Guida, K. Konishi, and H. Suzuki, Ann. Phys. (N.Y.) 249, 109 (1996).

[15] B. Bellet, P. Garcia, A. Neveu, Int. J. Mod. Phys. A 11, 5587
(1996); C. Arvanitis, F. Geniet, and A. Neveu, PM-94-19, hep-th/9506188; C. Arvanitis, F. Geniet, M. Iacomi, J. L. Kneur, and A. Neveu, Int. J. Mod. Phys. A 12, 3307 (1997).

[16] J. Glimm and A. Jaffe, Quantum Physics, A Functional Integral Point of View, 2nd ed. (Springer-Verlag, Berlin, 1987).

[17] G. H. Derrick, J. Math. Phys. 5, 1252 (1964).

[18] G. Auberson et al., Nuovo Cimento A 48, 1 (1978).

[19] W. E. Caswell, Ann. Phys. (N.Y.) 123, 153 (1979); I. G. Halliday and P. Suranyi, Phys. Lett. 85B, 421 (1979); Phys. Rev. D 21, 1529 (1981); J. Killingbeck, J. Phys. A 14, 1005 (1981); B. S. Shaverdyan and A. G. Ushveridze, Phys. Lett. 123B, 316 (1983); R. P. Feynman and H. Kleinert, Phys. Rev. A 34, 5080 (1986); R. Giachetti and V. Tognetti, Phys. Rev. Lett. 55, 912 (1985); Int. J. Magn. Magn. Mater. 54-57, 861 (1986); R. Giachetti, V. Tognetti, and R. Vaia, Phys. Rev. B 33, 7647 (1986); A. Okopinska, Phys. Rev. D 35, 1835 (1987); A. Duncan and M. Moshe, Phys. Lett. B 215, 352 (1988); C. M. Bender et al., Phys. Rev. D 37, 1472 (1988); A. Duncan and H. F. Jones, Nucl. Phys. B320, 189 (1989); H. F. Jones and M. Moshe, Phys. Lett. B 234, 492 (1990); I. Stancu and P. M. Stevenson, Phys. Rev. D 42, 2710 (1990); V. I. Yukalov, J. Math. Phys. 32, 1235 (1991); A. Neveu, Festschrift for A. Martin (Springer, Berlin, 1991); S. K. Gandhi et al., Nucl. Phys. B359, 429 (1991); A. N. Sissakian and I. L. Solovtsov, Z. Phys. C 54, 263 (1992).

[20] P. M. Stevenson, Phys. Rev. D 23, 2916 (1981).

[21] I. R. C. Buckley, A. Duncan, and H. F. Jones, Phys. Rev. D 47, 2554 (1993).

[22] R. Seznec and J. Zinn-Justin, J. Math. Phys. 20, 1398 (1979).

[23] Le Guillou and J. Zinn-Justin, Ann. Phys. (N.Y.) 147, 57 (1983). 